\title{
Response to the comment: Is observed upper airway obstruction patterns during drug-induced sedation endoscopy dose-dependent?
}

\author{
Michael Herzog ${ }^{1} \cdot$ Patrick Kellner $^{2}$
}

Received: 18 June 2017 / Accepted: 20 July 2017 /Published online: 7 September 2017

(C) Springer International Publishing AG 2017

Dear Editor,

The authors of the original article "Depth-dependent changes of obstruction patterns under increasing sedation during druginduced sedation endoscopy: results of a German monocentric clinical trial" (Sleep Breath., vol. 20, no. 3, pp. 1035-1043, 2016) would like to thank for this valuable letter to the editors.

The provided remarks confirm the main topics of the project. Initially, we clinically observed various patterns and degrees of collapsibility during the process of sedation during DISE. Based on this observation, the protocol of the study was created. As expected, we were able to demonstrate a depthdependent change of obstruction patterns. Of course, our results represent data which were obtained by our specific DISE protocol in our institution. In detail, we applied propofol by bolus, we used BIS for sedation monitoring, and we used a pictogram-based classification system which was introduced by us previously.

Certainly, DISE is and will most probably remain a highly individual procedure of investigating the upper airway.
Despite the effort of the European Working Group, a standardized procedure for DISE has not been proposed in the position paper on DISE. Probably, the update of the position paper will focus on this topic. On the other hand, it should be considered that a standardized procedure can hardly be established on base of the present available data on DISE. As long as we do not know which drug performs best, which depth of sedation is appropriate, which classification system provides the most adequate information, and which therapeutic consequences to draw from DISE results, we should not rely on a single DISE procedure. Personally, we believe that the procedure of DISE is still under change, seeking for an ideal way of performance. Of course, with any novel drug and DISE protocol, the process of evaluation and research will start from the beginning but will hopefully lead to more profound data on DISE. Until we reach a generally accepted consensus, several DISE protocols might coexist in parallel and hopefully, the scientific discussion will continue for the patient's benefit.
Michael Herzog

hno@ctk.de

1 Department of Otorhinolaryngology, Head and Neck Surgery, Carl-Thiem-Klinikum, Cottbus, Germany

2 Department of Anaesthesiology, University Medical Center Schleswig-Holstein, Ratzeburger Allee 16, 23538 Lübeck, Germany 